\title{
Reversible Metal-Insulator Transition in Ordered Metal Nanocrystal Monolayers Observed by Impedance Spectroscopy
}

\author{
Gil Markovich, ${ }^{1}$ Charles P. Collier, ${ }^{2}$ and James R. Heath ${ }^{1, *}$ \\ ${ }^{1}$ Department of Chemistry and Biochemistry, University of California, Los Angeles, 405 Hilgard Avenue, \\ Los Angeles, California 90095-1569 \\ ${ }^{2}$ Department of Chemistry, University of California, Berkeley, California 94720-1460
}

(Received 3 November 1997)

Low frequency impedance spectroscopy was applied on a Langmuir monolayer of alkylthiol passivated $35 \AA$ diameter silver quantum dots, as a function of interparticle separation distance. As interparticle spacing decreases below $30 \%$ of particle diameter, a reduction in interparticle charge tunneling time is observed. On further compression, the complex impedance of the films undergoes a transition from a parallel $R C$ equivalent circuit to an inductive circuit. Optical reflectance changes in the films are consistent with the deduced metal-insulator transition. [S0031-9007(98)05885-2]

PACS numbers: 71.30. $+\mathrm{h}, 73.20 . J \mathrm{c}$, 73.61.Tm

In recent years, techniques for preparing monodisperse, organically functionalized, metal quantum dots (QDs) have been developed [1]. Consequently, their crystallization to form superlattices and the study of structural and electronic properties of such ordered arrays are of growing interest [2-4]. One compelling feature of these superlattices is that three of the most critical parameters of a solidthe lattice site charging energy, the site-site coupling, and the lattice symmetry - may be independently varied. In this way, bulk electronic properties can be "designed" into the solid. For example, two dimensional, ordered superlattices of metal QDs can be prepared at the air/water interface on a Langmuir trough [5], and the Langmuir technique can be used to continuously vary the interparticle separation distance, thereby tuning the site-site coupling strength. In a recent publication from our laboratory, we showed that a Langmuir monolayer of alkylthiol passivated Ag quantum dots could be compressed reversibly through a metal-insulator transition under ambient conditions [6]. The deduced transition was based on monitoring the optical and nonlinear optical [second harmonic generation (SHG)] responses of the monolayer as the interparticle separation distance was reduced. A useful parameter for characterizing such monolayers is $D / 2 r$, where $D$ is interparticle separation, as measured between particle centers, and $r$ is particle radius. For $D / 2 r$ values $>1.3$, a classical coupling model could be employed to account for all experimental observations [7]. For $1.3>D / 2 r>1.2$, quantum coupling (i.e., exchange) appeared to dominate the trends in the linear and nonlinear optical responses. Below a $D / 2 r$ value of 1.2 , a sharp metal/insulator transition was observed. For the SHG response, the transition was observed as a sharp discontinuity in $\chi^{(2)}$. For the optical reflectance, the transition was observed as a sharp decrease in reflectivity, ultimately leading to a reflectance spectrum that matched that of a thin metal film. The transition could be reversed by expanding the barriers of the Langmuir trough.
In this paper, we utilize impedance spectroscopy (IS) to probe, at low frequencies, the charge transport mechanisms of the same films as they are compressed at the air-water interface. We find that, for $D / 2 r>1.3$, the film is well described as an equivalent $R C$ circuit. For $1.2<D / 2 r<$ 1.3 , we find that the characteristic time constant of the $R C$ circuit decreases rapidly with decreasing interparticle separation. Below $D / 2 r=1.2$, we find that the film undergoes a transition from an equivalent $R C$ circuit to an inductive circuit. These transport data are correlated with optical reflectance measurements for the same compression run, and the switch from a capacitive to an inductive circuit correlates with the reflectance signature that was ascribed previously to the metal-insulator transition [6].

IS has been used extensively to study localized conduction in solid electrolytes, ceramics [8], and disordered metal nanocrystal powders [9] as well as electrolyte solutions [10]. One important property of IS, as compared with simpler dc or ac conductivity measurements, is the ability to distinguish between electrode material (and other interface effects) and bulk responses, using different impedance-related complex functions [8]. This is especially important in our system, where it is necessary to separate the response of the film from that of the water subphase.

While the optical electromagnetic wave interaction with the silver nanocrystal films is dominated by the plasma oscillations of the free electrons in the particles, the radio frequency to the far-infrared regime should be more sensitive to long range conductivity. We therefore expect to observe transport signatures and characteristic time scales that correspond directly to the conduction electrons in the films.

In the present experiments, we prepare propanethiol passivated $35 \AA$ diameter silver QDs with a narrow $(<10 \%)$ size distribution as previously described [6]. After spreading the particles on the water subphase of a Langmuir trough, the barriers are slowly closed, compressing the 
nanocrystal monolayer. We measure the surface pressure isotherm as a function of film area, the complex admittance, and the optical reflectance of the system. We then correlate the optical signatures of the changes in the monolayer during compression to the low frequency admittance data. For the complex admittance measurements, two platinum wire electrodes were dipped into the film until just touching. Prior to the experiments, the electrodes were passivated with dodecylamine to make them hydrophobic, thus minimizing the water/electrode contact angle and contact area, and maximizing electrode-particle affinity.

The admittance was measured by a HP4284A $L C R$ meter in the frequency range $10 \mathrm{kHz}$ to $1 \mathrm{MHz}$. We have tried both two- and four-terminal configurations. Fourterminal measurements could not yield reliable phase information since the interface impedance at the current terminals was much higher than the bulk film impedance. The results presented in this paper were therefore taken using a two-electrode setup, at frequencies above $10 \mathrm{kHz}$, where the water double layer response is small. Prior to particle spreading, we record the pure water contribution to the admittance.

For the optical measurements, a fiber coupled, UVvisible diode array spectrometer is used, as in Ref. [6], to record reflectance spectra of the film at a $35^{\circ}$ angle of incidence as the film is compressed. Typically, 30 spectra are recorded during a compression run, and correlated to both the film state as inferred by the pressure-area isotherms, and to changes in the complex admittance.

In the data analysis, we assume that the nanocrystal film complex admittance $\left(Y^{*}\right)$ is separable from the measured total admittance since we are only interested in changes in the impedance spectrum with decreasing interparticle separation. Following this, we model the water/film system as two parallel admittance elements. This assumption should be valid in our measurement frequency range $(10 \mathrm{kHz}-1 \mathrm{MHz})$, where there is no significant interference from the water double layer at the electrodes, and charge transfer at the water-particle interface is improbable. A second assumption, which is general to most IS experiments, is linear response. We therefore keep the excitation voltage $\leq 200 \mathrm{mV}$.

After subtracting the water contribution from the complex admittance signal, the data are transformed to different impedance-related functions such as the complex impedance

$$
Z^{*}=\frac{1}{Y^{*}}
$$

and complex dielectric modulus

$$
M^{*}=j C_{0} \omega Z^{*},
$$

where $j=\sqrt{-1}, C_{0}$ is the geometric capacitance of the cell defined by the electrodes and film, and $\omega$ is the angular frequency. The dielectric modulus is the inverse of the more familiar complex dielectric permittivity. The complex dielectric modulus is a particularly useful functional form to plot the impedance data when conductive systems with relatively low capacitance are studied [11]. Metal nanoparticles in this size regime have typically a capacitance of the order of $10^{-19}$ to $10^{-18} \mathrm{~F}$ per particle [12]. We present our results in the complex modulus plane in Fig. 1(a).

Figure 1(b) shows the compression isotherm for the same experiment. Points corresponding to the curves plotted in Fig. 1(a) are indicated. We have previously employed transmission electron microscopy to extensively characterize Ag quantum dot monolayers [2,5,6]. We have reported on how various parameters such as ligand size,
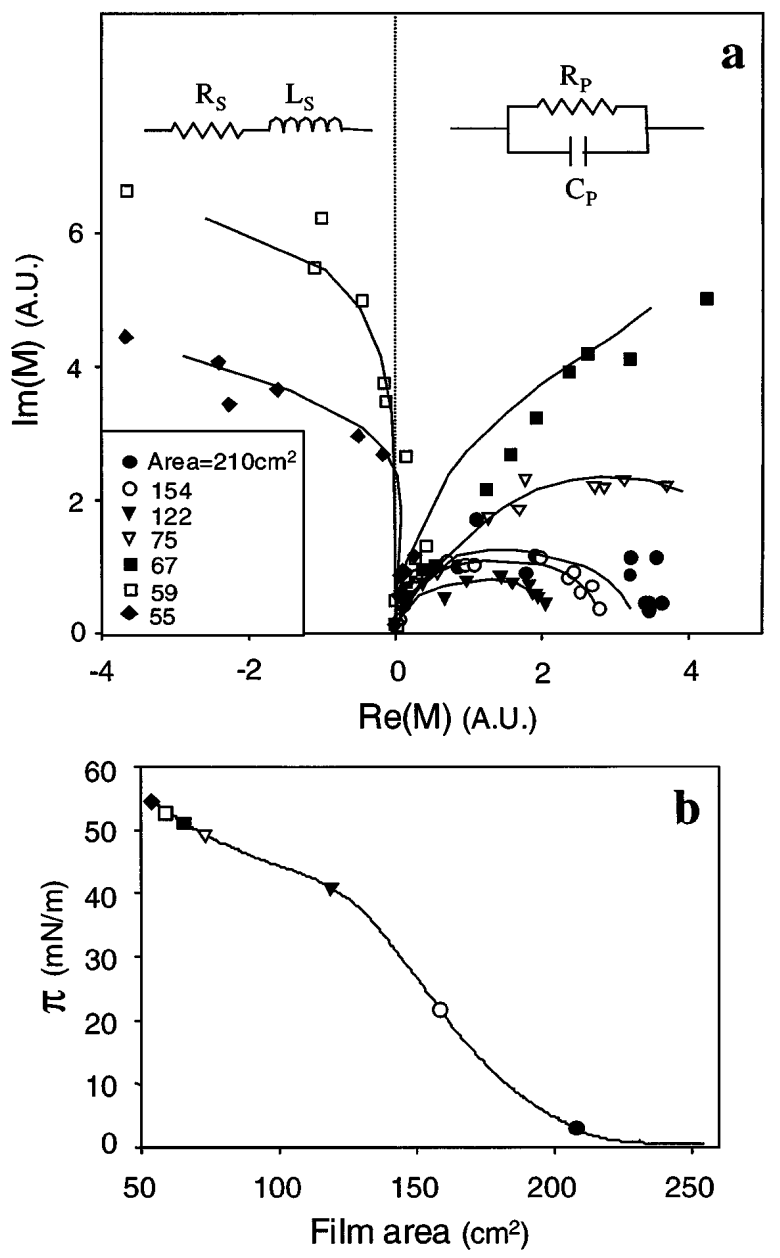

FIG. 1. (a) Plot of the frequency dependent dielectric modulus, in the complex plane, for a monolayer of $3.5 \mathrm{~nm}$ diameter, propanethiol capped $\mathrm{Ag}$ quantum dots. The particle film response is characterized initially by an $R C$ equivalent circuit, in conformity with a picture of localized conductance. As the particles are compressed to a separation of less than $6 \AA$, the film becomes inductive, indicating the presence of metalliclike transport in the film. (b) The Langmuir isotherm of the monolayer compression, with labels corresponding to the various curves in the top graph. Although the isotherm indicates a collapse near $130 \mathrm{~cm}^{2}$ film area, the collapse is heterogeneous, occurring only at the mobile barriers. The central region of the film, where transport measurements are carried out, remains an uncollapsed monolayer. 
particle diameter, and monolayer compression correlate to interparticle separation distance, and how interparticle separation distance correlates to the optical properties of the films [7]. Based on those studies, and the optical and isotherm data for the present experiment, we estimate a distance between the surfaces of the metal cores $\delta=10 \AA$ for the continuous, uncompressed monolayer $(D / 2 r=$ $1.3)$ and $\delta=5 \AA$ at full compression $(D / 2 r=1.14)$.

At low compression, we observe distorted semicircles in the complex modulus plane. The system can be represented by an equivalent parallel $R C$ circuit [13], as in the simple Debye relaxation model [14], where $\tau=$ $R C$ is the dielectric relaxation time. Disordered systems typically are better described by a distribution of $R C$ values [13]. Since our particle films are reasonably well ordered, we expect a narrower distribution of relaxation times. Nevertheless, the distortion in the semicircles in Fig. 1(a) indicates that there is a superposition of at least two semicircles that leads to the observed curve, indicating at least two different time scales influencing the response. We therefore fitted the curves in Fig. 1(a) using the relations

$$
\begin{array}{r}
M^{\prime}=A_{i} \frac{\left(\omega \tau_{i}\right)^{2}}{1+\left(\omega \tau_{i}\right)^{2}}, \quad M^{\prime \prime}=A_{i} \frac{\omega \tau_{i}}{1+\left(\omega \tau_{i}\right)^{2}}, \\
i=1,2,
\end{array}
$$

where $M^{\prime}$ and $M^{\prime \prime}$ are the real and imaginary components of the dielectric modulus, respectively. The amplitudes $A_{1,2}$ and the conductivity relaxation times $\tau_{1,2}$ are free parameters. The fits are included in Fig. 1(a) as lines.

The slower fitted relaxation time $\tau_{2}$ and its amplitude $A_{2}$ are nearly independent of compression. We conclude that this component represents an interface effect, probably representative of an electrode-particles $R C$ equivalent circuit. In Fig. 2, we present the change only in the characteristic bulk film parameters, $\tau_{1}$ and $A_{1}$, as the film area decreases.

The bulk relaxation time decreases sharply at the last part of the compression, as its relative contribution increases. The increase in semicircle radii along compression in Fig. 1(a) is due to an increasing $A_{1}$. These radii are proportional to $1 / C$. Based on the behavior of the radii, there is a slight increase in capacitance at the beginning of compression, as the film becomes continuous (percolating).

$\tau_{1}$ begins to decrease below a film area of about $120 \mathrm{~cm}^{2}$. As the film is compressed from an area of 120 to $55 \mathrm{~cm}^{2}$, there is a very small decrease in interparticle separation. Although the isotherm shows a slope change that is indicative of monolayer collapse near $130 \mathrm{~cm}^{2}$, visual inspection revealed that the collapse was heterogeneous, occurring only at the mobile barriers. Most of the reduced film area is due to particle buildup at the barriers. In the central region of the film, where the optical and electrical properties are probed, there is no collapse. We estimate from the details of the isotherm, coupled with

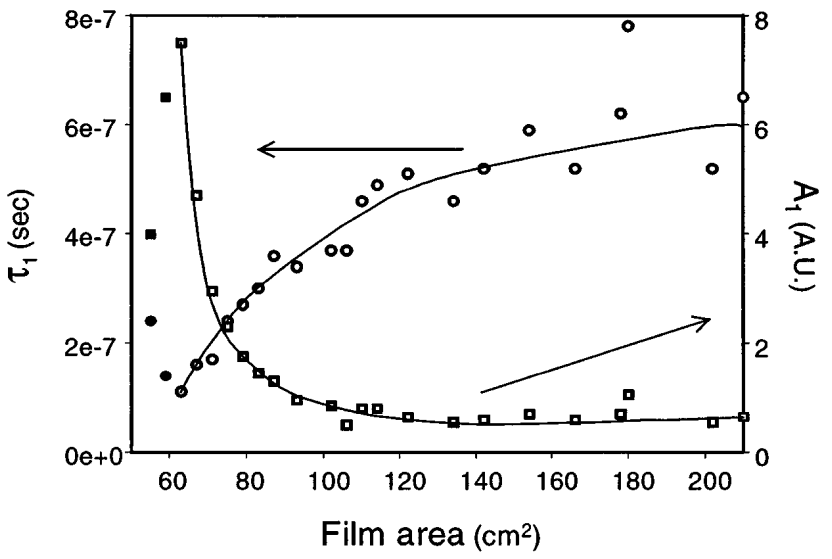

FIG. 2. A plot of relative amplitude $A_{1}$ (squares) and characteristic time constant $t_{1}$ (circles) of the dielectric modulus of the QD monolayer. As the monolayer is compressed, the time constant decreases, possibly corresponding to a faster electron tunneling rate. Below about $65 \mathrm{~cm}^{2}$ film area, the time constant appears to invert. In fact, this is the point where the film becomes inductive, and we are no longer able to extract a meaningful time constant.

previous probes of similar films with electron microscopy, that between 120 and $55 \mathrm{~cm}^{2}$ there is a change of only a few percent in $D / 2 r$.

Our fit of $M^{*}$ for the plots in Fig. 1(a) to capacitive tunnel junctions becomes unreliable below a film area of $70 \mathrm{~cm}^{2}$. At this point appears the most striking feature: The real part of $M^{*}$ becomes negative. Such a situation is not possible within the parallel $R C$ model [Eq. (3)], and argues that the film has become inductive [top left of Fig. 1(a)].

If we assume that we have an insulator-metal transition at a film area near $60 \mathrm{~cm}^{2}$, then we should have macroscopic metallic conduction pathways through the film and the capacitive behavior of the tunneling current should be replaced by the dominating inductive impedance of a metal film. The inductive current is $180^{\circ}$ off the capacitive current phase, and should therefore contribute negatively to the real part of $M^{*}$. We therefore use a series $R_{S} L_{S}$ equivalent circuit to represent the film at the last part of compression, using

$$
M^{\prime}=-C_{0} L_{S} \omega^{2}, \quad M^{\prime \prime}=C_{0} L_{S} \frac{\omega}{\tau_{L}},
$$

where $\tau_{L}=L_{S} / R_{S}$.

The two fitted curves for area $=59,55 \mathrm{~cm}^{2}$ shown in Fig. 1(a) are superpositions of one parallel $R C$ circuit (representing the interface contribution $-\tau_{2}, A_{2}$ ) and one series $R_{S} L_{S}$ circuit. Note that the fit is only qualitative, since we can obtain only the ratio $C_{0} L_{S} / \tau_{L}$ in the fit, without resolving its individual contributions. The $R C$ contribution to the fit is negligible at this point and was included for consistency with the low compression curves.

Two optical observations lend support to the insulatormetal transition picture. A set of reflectance spectra 
collected simultaneously to the impedance measurements presented in Fig. 1(a) is essentially the same as shown in Fig. 4 of Ref. [6]. The reflectance spectra, showing the particle plasmon resonance feature, increases in intensity at the initial stages of compression. As the film area is decreased below $120 \mathrm{~cm}^{2}$, the reflectance starts to drop. With continued compression, the particle plasmon resonance is eventually lost, and the reflectance spectrum will match that of a thin metal film.

The second observation was that, for area $<70 \mathrm{~cm}^{2}$, we could observe, at normal incidence, the film attaining a shiny, silver metallic luster which, for certain of the films, spread homogeneously over the film area. The appearance and disappearance of the luster is reversible, as are the changes in the $45^{\circ}$ reflectance spectra.

As interparticle separation is reduced, the data suggest that the mechanism of charge transport changes from hopping to tunneling to metallic transport. "Hopping" conductivity and tunneling are both characterized by an energy barrier, but with little or no energy difference between the initial and final states. When the film becomes continuous (percolating) around $200 \mathrm{~cm}^{2}$, thermally activated hopping conductivity is the dominant transport mechanism, and tunneling is a relatively minor component [4]. Between 200 and $120 \mathrm{~cm}^{2}(1.3>D / 2 r>$ $1.2)$, there is very little change in the complex modulus plots, indicating that the conductivity mechanism does not change. In the range $120-65 \mathrm{~cm}^{2}(D / 2 r<1.2)$, the characteristic time constant $\tau_{1}$ of the $R C$ circuit begins to decrease. One interpretation is that a decreasing $\tau_{1}$ represents an increasing tunneling rate between single particles. An alternative explanation is that there is sufficient charge delocalization within the film that this rate represents variable range hopping of electrons between more extended domains. Whatever the case, the complex modulus plots that correspond to this region of the isotherm are consistent with individual particles coupled to one another through an $R C$ circuit, such as that shown at the top right of Fig. 1(a). Below $65 \mathrm{~cm}^{2}$, however, this picture of individual particles is no longer valid, as the circuit has become inductive. This is consistent with metallic conductivity pathways. This change from a transport picture based on single particles to one based on a metallic film is analogous to what is observed in the reflectance spectra in which the plasmon resonance signature of a spherical silver particle gives way to the reflectance spectrum of a bulk film.

The role of order-disorder to the metal-nonmetal transition for our system of nanoparticles is not clear. We have investigated monolayers that are characterized by broad particle size distributions, and, although the reflectance spectrum of such films indicates strong dipole-dipole coupling effects, we have never observed such films to undergo a metal-insulator transition. Nevertheless, even our "best" films exhibit a certain amount of disorder. The (Coulombic) site-charging energy $\left(\varepsilon_{c}\right)$ that characterizes our "artificial atoms" of silver QDs is large $\left(\varepsilon_{c} \sim 0.2 \mathrm{eV}\right)$ relative to the expected single electron energy level spacing within a single particle $(\sim 5 \mathrm{meV})$, and makes our system unique within the context of metal-insulator transitions. $\varepsilon_{c}$ may also be large in comparison to energies that characterize the disorder in this system, such as particle size distributions and variations in local chemical environments. As such, it may effectively exclude a disorder driven, Anderson-type transition from our system [15]. Since $\varepsilon_{c} \gg k T$ at room temperature, this system should be well below the critical temperature for the existence of a first order metal-insulator transition. This would constitute a Mott transition [16,17] under ambient conditions, which would be most exciting. Hall effect and microwave dielectric measurements are currently being carried out in order to further test these possibilities.

We thank R.S. Williams and Hewlett Packard Corporation for loan of the LCR meter used in these experiments. We acknowledge helpful discussions with Steve Kivelson, Rich Saykally, and Alex Bratkovski. J. R. H. and G. M. acknowledge support from an NSF-NYI grant. C.P.C. acknowledges support from NSF Grant No. CHE-9424482.

*Author to whom all correspondence should be addressed. [1] M. Brust et al., J. Chem. Soc. Chem. Commun. 801 (1994).

[2] P. C. Ohara, D. V. Leff, J. R. Heath, and W. M. Gelbart, Phys. Rev. Lett. 75, 3466 (1995).

[3] S. A. Harfenist et al., Adv. Mater. 9, 817 (1997).

[4] R. P. Andres et al., Science 273, 1690 (1996).

[5] J. R. Heath, C. M. Knobler, and D. V. Leff, J. Phys. Chem. 101, 189 (1997).

[6] C.P. Collier, R. J. Saykally, J. J. Shiang, S. E. Henrichs, and J. R. Heath, Science 277, 1978 (1997).

[7] J. J. Shiang, C.P. Collier, R. J. Saykally, and J. R. Heath, J. Phys. Chem. (to be published).

[8] J. R. Macdonald, Impedance Spectroscopy (Wiley, New York, 1987).

[9] U. Simon, G. Schon, and G. Schmid, Angew. Chem., Int. Ed. Engl. 32, 250 (1993).

[10] M. Sluyters-Rehbach and J. H. Sluyters, in Comprehensive Treatise of Electrochemistry, edited by E. Yeager et al. (Plenum, New York, 1984), Chap. 4.

[11] I. M. Hodge, M. D. Ingram, and A. R. West, J. Electroanal. Chem. 74, 125 (1976).

[12] See, for example, R. P. Andres et al., Science 272, 1323 (1996).

[13] R. Gerhardt, J. Phys. Chem. Solids 55, 1491 (1994).

[14] P. J.W. Debye, Polar Molecules (Dover, New York, 1960).

[15] See P. A. Cox, The Electronic Structure and Chemistry of Solids (Oxford University, Oxford, 1987), p. 222.

[16] N. F. Mott, Metal-Insulator Transitions (Taylor \& Francis, London, 1990).

[17] C. A. Stafford and S. Das Sarma, Phys. Rev. Lett. 72, 3590 (1994). 